\title{
Correlation of a feline muscle mass score with body composition determined by dual-energy $\mathrm{X}$-ray absorptiometry
}

\author{
Kathryn E. Michel ${ }^{1 *}$, Wendy Anderson ${ }^{2}$, Carolyn Cupp ${ }^{2}$ and Dorothy P. Laflamme ${ }^{2}$ \\ ${ }^{1}$ Department of Clinical Studies-Phila, School of Veterinary Medicine, University of Pennsylvania, 3900 Delancey Street, \\ Philadelphia, PA 19104-6010, USA \\ ${ }^{2}$ Nestlé Purina Pet Care Product Technology Center, Saint Joseph, MO, USA
}

(Received 15 October 2010 - Accepted 11 January 2011)

\section{Abstract}

Body condition scoring (BCS) systems primarily assess body fat. Both overweight and underweight animals may have loss of lean tissue that may not be noted using standard BCS systems. Catabolism of lean tissue can occur rapidly, may account for a disproportionate amount of body mass loss in sick cats and can have deleterious consequences for outcome. Therefore, along with evaluation of body fat, patients should undergo evaluation of muscle mass. The aims of the present study were first to evaluate the repeatability and reproducibility of a 4-point feline muscle mass scoring (MMS) system and second to assess the convergent validity of MMS by dual-energy X-ray absorptiometry (DXA). MMS was as follows: 3, normal muscle mass; 2, slight wasting; 1, moderate wasting; 0, severe wasting. For the first aim, forty-four cats were selected for evaluation based on age and BCS, and for the second aim, thirty-three cats were selected based on age, BCS and MMS. Cats were scored by ten different evaluators on three separate occasions. Body composition was determined by DXA. Inter- and intra-rater agreement were assessed using kappa analysis. Correlation between MMS and BCS, age, percentage lean body mass and lean body mass (LBM) was determined using Spearman's rank-order correlation. The MMS showed moderate inter-rater agreement in cats that scored normal or severely wasted $(\kappa=0.48-0.53)$. Intra-rater agreement was substantial $(\kappa=0 \cdot 71-0 \cdot 73)$. The MMS was significantly correlated with BCS $(r 0.76, P<0.0001)$, age $(r-0.75, P<0.0001)$, LBM $(\mathrm{g})(r 0.62, P<0 \cdot 0001)$ and percentage LBM $(r-0.49, P<0 \cdot 0035)$. Additional investigation is needed to determine whether the MMS can be refined and to assess its clinical applicability.

\section{Key words: Body Condition Scoring: Lean Body Mass: Dual-energy X-ray Absorptiometry: Cats}

There has been growing acceptance of the use of body condition scoring (BCS) as a component of nutritional assessment for companion animals. This technique can be a very useful tool, particularly when dealing with obese patients or when educating people about proper feeding management for their pets. However, there are some limitations with the present scoring systems when dealing with patients who are undergoing catabolism associated with injury or disease. This is because these scoring systems focus on the assessment of body fat through evaluation of the body silhouette and palpation of adipose tissue ${ }^{(1)}$. Wasting of muscle mass is only assessed in significantly underweight individuals. However, both underweight and overweight patients can undergo catabolism of lean body mass (LBM) that may not be recognised using a standard BCS system.

Catabolism of LBM can occur rapidly, particularly in critically ill patients, and may account for a disproportionate amount of body mass lost in ill patients ${ }^{(2-4)}$. This loss of lean tissue is important to recognise because, while the purpose of the adipose tissue is to serve as an energy reserve, there is no analogous reserve of endogenous protein. Virtually all endogenous proteins are serving some function, and, consequently, continued catabolism will eventually have deleterious consequences for the patient ${ }^{(5)}$.

Therefore, the process of body condition assessment should include not only the standard evaluation of body silhouette and adipose tissue as a determinant of energy reserves, but also a separate evaluation of muscle mass as an estimate of lean tissue status. There has been growing acceptance of the need to assess muscle mass both in the clinical setting and for investigational purposes ${ }^{(6-8)}$; however, to our knowledge, no one has ever validated a muscle mass scoring (MMS) system in companion animals.

The specific aims of the present investigation were first to evaluate the repeatability and reproducibility of a 4-point feline MMS system and second to assess the convergent validity (predictability) of this scoring system as it applies to cats by dual-energy X-ray absorptiometry (DXA).

Abbreviations: BCS, body condition scoring; DXA, dual-energy X-ray absorptiometry; LBM, lean body mass; MMS, muscle mass scoring.

*Corresponding author: K. E. Michel, fax +1 215573 6050, email michel@vet.upenn.edu 
Table 1. Description of the muscle mass scoring system

\begin{tabular}{ll}
\hline Score & Muscle mass \\
\hline 0 & $\begin{array}{c}\text { On palpation over the spine, scapulae, skull or wings } \\
\text { of the ilia, muscle mass is severely wasted } \\
\text { On palpation over the spine, scapulae, skull or wings } \\
\text { of the ilia, muscle mass is moderately wasted } \\
\text { On palpation over the spine, scapulae, skull or wings } \\
\text { of the ilia, muscle mass is mildly wasted } \\
\text { On palpation over the spine, scapulae, skull or wings } \\
\text { of the ilia, muscle mass is normal }\end{array}$ \\
\hline
\end{tabular}

\section{Experimental methods}

The study was performed at the Nestlé Purina Pet Care Product Technology Center (St Joseph, MO, USA), with review and approval by the Institutional Animal Care and Use Committee. For the first specific aim, forty-four cats were selected to represent a wide range in age (1-15 years) and body condition (BCS 3-8/9). Cats were categorised by age as young ( $<6$ years), middle-aged $(6-10$ years) and elderly ( $>10$ years), and by BCS as underweight (BCS $<4 / 9)$, good body condition (4-6/9) or overweight ( $>6 / 9)$. At least four cats were selected to represent each (age $\times$ BCS) category. A total of ten veterinarians and veterinary technicians received hands-on training on how to use the MMS, and then each individual evaluated and scored all forty-four cats on three separate occasions. The MMS system is described in Table 1. The order of evaluation of the cats was randomised among scorers and for each occasion of scoring for each evaluator. The three separate evaluations took place at least 1 week apart.

For the second specific aim, thirty-three cats were purposely selected for evaluation based on age (3-17 years), BCS (2-7/9) and MMS (0-3) in an attempt to generate a population that represented the scope of each of the three parameters, although it was not possible to find cats that were representative of all possible permutations. For example, within this population of healthy cats, there were no cats classified as underweight that were assessed as having normal muscle mass nor young cats with significant muscle wasting. Each cat had body composition determined by DXA.

DXA scans were performed with cats under general anaesthesia using a GE Lunar Prodigy DXA machine (EnCore 2006 version 10.51.006 software, Lunar Corporation, Madison, WI, USA). Cats were maintained in dorsal recumbency and scanned in total body (Human) model, under the thin $(<13 \mathrm{~cm})$ setting.

\section{Statistical analysis}

Inter-rater agreement (reproducibility) and intra-rater agreement (repeatability) were assessed using the $\kappa$ statistic. $\kappa$ statistics are interpreted as fair (0.21-0.40), moderate (0.41-0.60), substantial $(0 \cdot 61-0 \cdot 80)$ or almost perfect $(0 \cdot 81-1 \cdot 0)$ agreement. The correlation between the MMS and BCS, age (years), LBM (g) and percentage LBM was determined using Spearman's rank-order correlation. All analyses were performed using commercially available software (Stata version 8; StataCorp, College Station, TX, USA).

\section{Results}

Using the median of all thirty scores that each cat received, eleven cats were scored as having normal muscle mass, fourteen were scored as having mild muscle wasting, eleven were scored as having moderate muscle wasting and eight were scored as having severe muscle wasting. Inter-rater agreement (reproducibility) was moderate (all $P$ values $<0 \cdot 001$ ) on each of the three occasions of evaluation for the cats that scored normal $(0.48,0.51$ and 0.53$)$ or severely wasted $(0.49,0.50$ and 0.59). However, inter-rater agreement was only fair (all $P$ values $<0 \cdot 001)$ for the intermediate scores $(0 \cdot 25,0 \cdot 20$, 0.26 and $0.23,0.31,0.31$ for cats that scored mildly and moderately muscle-wasted, respectively). Intra-rater agreement (repeatability) was substantial (all $P$ values $<0.001$ ) whether for the first $v$. the second ratings $(0.73)$ or the second $v$, the third ratings $(0 \cdot 71)$.

The MMS was significantly and positively correlated with BCS $(r 0.76, P<0.0001)$ and LBM (g) $(r$ 0.62, $P<0.0001)$, and was negatively correlated with age $(r-0.75, P<0.0001)$ and percentage LBM $(r-0.49, P<0.0035)$. The regression plots for the MMS $v$. LBM (g) and MMS $v$, age are shown in Fig. 1.

\section{Discussion}

The feline MMS was found to have substantial repeatability but only fair-to-moderate reproducibility. The intermediate scores for mild and moderate muscle wasting had the least reproducible scores, and it is possible that a 3-point scoring
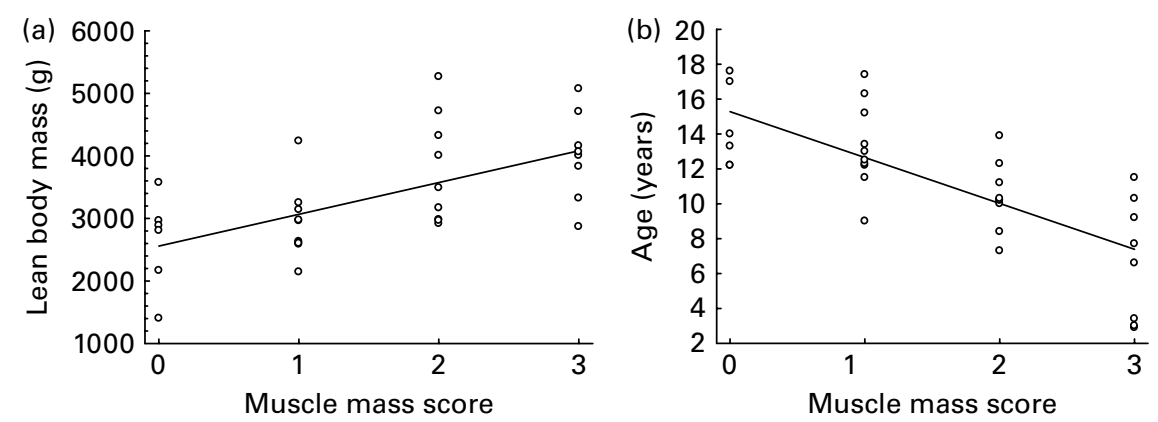

Fig. 1. Association between (a) muscle mass score and lean body mass in $\mathrm{g}(y=2557.28+507 \cdot 78 X ; r 0.62, P<0 \cdot 0001)$ and (b) muscle mass score and years of age $(y=15.25-2.63 X ; r=-.75, P<0.0001)$. 
system that would combine mild-to-moderate wasting as a single score may perform better. Another refinement of the MMS would be to focus on the evaluation of one portion of the musculature, for example the epaxial muscles of the spine, rather than the four different regions that are included in the current scoring system. Additional evaluations would be needed to determine whether such modifications would enhance the functionality of the MMS system.

It is not unexpected that the correlation between the MMS and LBM as determined by DXA was modest. Skeletal muscle is the only one component of LBM, which also includes visceral organs and typically accounts for approximately $35-40 \%$ of $\mathrm{LBM}^{(9)}$. The lack of a stronger correlation with LBM does not necessarily diminish the utility of the MMS for clinical patients. In starvation concurrent with metabolic stress, the majority of protein catabolism involves skeletal muscle proteins ${ }^{(10)}$, so with regard to assessing the impact of the catabolic state on the nutritional status of a patient, assessment of muscle wasting may have the most prognostic significance. Markers of depletion of LBM in critically ill human patients have been found to be associated with patient outcomes ${ }^{(5,11)}$.

With regard to the seemingly counter-intuitive negative correlation between the MMS and percentage LBM, it is necessary to take into account that the percentage LBM will be affected by the adiposity of the patient as well as by the absolute amount of lean tissue. In the present study, the relationship of percentage LBM to MMS was confounded by the fact that both underweight and overweight cats were included in the study population.

A shortcoming of the present study is that it was performed in healthy cats of a wide range of ages whose loss of LBM was generally attributed to normal age-related changes rather than disease. It is likely that muscle wasting differs somewhat when induced by disease.

In conclusion, individuals can apply a 4-point feline MMS system with substantial repeatability. However, the reproducibility of this system among evaluators was not as strong, especially for discriminating among scores for cats that had mild-to-moderate loss of muscle mass. There was fair correlation between the feline MMS and absolute LBM as determined by DXA. However, additional investigation is desirable to determine whether this MMS system can be further refined particularly in terms of reproducibility and to assess its clinical applicability.

\section{Acknowledgements}

K. E. M. received research funding from the Nestlé Purina PetCare Company and serves on an Advisory Council for that company. Funding for the present investigation was from the Nestlé Purina PetCare Company. All authors were involved in the design and execution of the study and the data analysis. K. E. M. was the principal author of the manuscript, with review and input from the other authors.

\section{References}

1. Laflamme DP (1997) Development and validation of a body condition score system for cats: a clinical tool. Feline Pract 25, 13-18.

2. Blackburn GL, Bistrian BR, Maini BS, et al. (1977) Nutritional and metabolic assessment of the hospitalized patient. JPEN J Parenter Enteral Nutr 1, 11-22.

3. Michel KE, King LG \& Ostro E (1997) Measurement of urinary urea nitrogen as an estimate of total urinary nitrogen loss in dogs. J Am Vet Med Assoc 210, 356-359.

4. Biourge V, Groff JM, Morris JG, et al. (1994) Long-term voluntary fasting in adult obese cats: nitrogen balance, plasma amino acids concentrations and urinary orotic acid excretion. J Nutr 124, 2680S-2682S.

5. Ravasco P, Camilo ME, Gouveia-Oliveira A, et al. (2002) A critical approach to nutritional assessment in critically ill patients. Clin Nutr 21, 73-77.

6. Baldwin K, Bartges JW, Buffington CAT, et al. (2010) AAHA nutritional assessment guidelines for dogs and cats. $J \mathrm{Am}$ Anim Hosp Assoc 46, 285-296.

7. Michel KE, Sorenmo K \& Shofer FS (2004) Evaluation of body condition and weight loss in dogs presenting to a veterinary oncology service. $J$ Vet Intern Med 18, 692-695.

8. Baez JL, Michel KE, Sorenmo K, et al. (2007) A prospective investigation of cachexia in feline cancer patients and its prognostic significance. J Feline Med Surg 9, 411-417.

9. Helmsfield SB, McManus CB, Seitz SB, et al. (1984) Anthropometric assessment of adult protein energy malnutrition. In Nutritional Assessment, pp. 28-82 [RA Wright and SB Helmsfield, editors]. London: Blackwell Scientific Publications, Inc.

10. Duff JH, Viidik T, Marchuk JB, et al. (1979) Femoral arteriovenous amino acid differences in septic patients. Surgery $\mathbf{8 5}$, 344-348.

11. Sungurtekin H, Sungurtekin U, Ozlem O, et al. (2008) Nutrition assessment in critically ill patients. Nutr Clin Pract $\mathbf{2 3}$ 635-641. 